\title{
(a) OPEN ACCESS \\ Sonic hedgehog signaling pathway promotes pancreatic cancer pain via nerve growth factor
}

\author{
Liang Han, ${ }^{1}$ Jie Jiang, ${ }^{2}$ Mengwen Xue, ${ }^{3}$ Tao Qin, ${ }^{1}$ Ying Xiao, ${ }^{1}$ Erxi Wu, ${ }^{4,5}$ Xin Shen, ${ }^{3}$ \\ Qingyong $\mathrm{Ma}^{1}$ Jiguang $\mathrm{Ma}^{3}$
}

- Additional material is published online only. To view please visit the journal online (http://dx.doi.org/10.1136/ rapm-2019-100991).

${ }^{1}$ Department of Hepatobiliary Surgery, Xi'an Jiaotong University Medical College First Affiliated Hospital, Xi'an, Shaanxi, China

${ }^{2}$ Department of Medical Oncology, Shaanxi Provincial People's Hospital, Xi'an, China ${ }^{3}$ Department of Anesthesiology, Xi'an Jiaotong University Medical College First Affiliated Hospital, Xi'an, China

${ }^{4}$ Department of Surgery, Baylor Scott and White Health, Dallas, Texas, USA

Department of Neurosurgery, Baylor Scott and White Health, Dallas, Texas, USA

\section{Correspondence to}

Dr Qingyong Ma, Department of Hepatobiliary Surgery, Xi'an Jiaotong University Medical College First Affiliated Hospital, Xi'an, China;

qyma56@xjtu.edu.cn

$\mathrm{LH}$ and $\mathrm{JJ}$ are joint first authors.

Received 12 September 2019 Revised 30 October 2019 Accepted 3 November 2019 Published Online First

1 December 2019
Check for updates

(C) American Society of Regional Anesthesia \& Pain Medicine 2020. Re-use permitted under CC BY. Published by BMJ.

\section{To cite: Han $L$,}

Jiang J, Xue M, et al.

Reg Anesth Pain Med

2020:45:137-144

\section{ABSTRACT}

Background Many patients with pancreatic cancer (PC) suffer from abdominal pain and back pain. However, the cause of pain associated with PC is largely unclear. In this study, we tested the potential influence of the sonic hedgehog $(\mathrm{sHH})$ signaling pathway on PC pain.

Methods Substance P (SP) and calcitonin gene-related peptide (CGRP) expression was measured in cultured PC cells and dorsal root ganglions (DRG) by real-time PCR, western blotting analysis and ELISA. Small interfering RNA transfection and plasmid constructs were used to regulate the expression of $\mathrm{sHH}$ in the AsPc-1 and Panc-1 cell lines. Pain-related behavior was observed in an orthotopic tumor model in nude mice.

Results In this study, the results show that $\mathrm{sHH}$ increased the expression of SP and CGRP in DRGs in a concentration and time-dependent manner. Additionally, sHH secretion from PC cells could activate the sHH signaling pathway and, in turn, increase the expression of nerve growth factor (NGF), P75, and TrkA in DRGs. Furthermore, the $\mathrm{SHH}$ signaling pathway and NGF/NGF receptor contributed to pain sensitivity in a nude mouse model.

Conclusion Our results demonstrate that $\mathrm{PC}$ pain originates from the $\mathrm{SHH}$ signaling pathway, and NGF mediates the pain mechanism via regulating SP and CGRP.

\section{INTRODUCTION}

Pancreatic cancer (PC) is one of the most aggressive malignant human cancers and is associated with an extremely poor prognosis. ${ }^{12}$ Patients with PC often experience abdominal and back pain. ${ }^{3}$ Many physiopathologic mechanisms are involved in PC pain, for example, nerve damage, inflammation, and perineural invasion (PNI). ${ }^{4-7}$ PNI is associated with poor survival, local recurrence, and neuropathic pain. Currently, pain is one of the major outcomes of the interaction between nerves and PC. ${ }^{89}$

Recent studies have demonstrated that expression of the sonic hedgehog $(\mathrm{sHH})$ ligand contributes to PC progression. ${ }^{10}$ In addition, paracrine sHH signaling plays an important role in communication among the tumor, stroma, and nerves. sHH signaling is initiated by the binding of the sHH ligand to Patched-1 (PTCH1), which relieves repression of the transducer protein Smoothened (SMO), thus activating the GLi family of transcription factors. The activation of downstream factors such as GLi-1 and GLi-2 regulates target gene expression, resulting in proliferation, cell survival, and angiogenesis. ${ }^{11}$ As reported previously, sHH regulates nociceptive sensitization and guides the spatial pathfinding of raphespinal tract axons. ${ }^{12}$

Nerve growth factor (NGF) is a neurotrophic factor that belongs to the family of neurotrophins. NGF can bind tyrosine receptor kinases (Trk) and $\mathrm{p} 75$, a member of the tumor necrosis factor receptor superfamily, to promote neuronal survival and axonal growth. In the last decade, NGF and TrkA have been recognized as important mediators of pain. ${ }^{13-15}$ Additionally, NGF and TrkA are associated with the neuronal expression of inflammatory neuropeptides such as calcitonin gene-related peptide (CGRP) and substance $\mathrm{P}(\mathrm{SP}) .^{9} 16$

$\mathrm{sHH}$ signaling is directly involved in nerve cell activity and the regulation of PC progression. ${ }^{17} 18$ Some evidence strongly suggests that NGF and its receptors play a role in neuropathic pain. ${ }^{13} 1519$ In this study, we demonstrate that PC pain originates from the sHH signaling pathway in nerves and that neurotrophic factors mediate the pain mechanism by regulating SP and CGRP.

\section{MATERIALS AND METHODS}

Orthotopic transplantation of tumor cells into nude mice

Prepared PC cells were injected into the pancreas of nude mice exposed by midline laparotomy $\left(20 \mu \mathrm{L}\right.$ of cells at a total concentration of $1 \times 10^{6} \%$ $\mu \mathrm{L}, 4-6$ sites; $20 \mu \mathrm{l}$ total volume). After 4 weeks, the carcinoma model was successfully generated, and metastases to different locations did not occur by ultrasonic testing. Then, drug treatment and pain behavior could be tested.

\section{Von Frey filament testing}

After shaving the abdomens of nude mice, the abdomen of each mouse was stimulated 20 times at different intensities. Each stimulation lasted $2 \mathrm{~s}$ with an approximately $30 \mathrm{~s}$ interval between stimulations. A positive response consisted of lifting the belly and/or scratching or licking the abdomen. The number of responses was summed over all 20 stimulations. Each group had 10 mice for the testing.

\section{Cell culture and reagents}

The human PC cell lines AsPc-1 and Panc-1 were obtained from the American Type Culture Collection (Manassas, VA) and cultured in Dulbecco's Modified Eagle Medium (DMEM) supplemented with 10\% fetal bovine serum (FBS) and 1\% antibiotics/antimycotics in a humidified $5 \% \mathrm{CO}_{2}$ atmosphere at $37^{\circ} \mathrm{C}$. Antibodies against $\mathrm{sHH}, \mathrm{SMO}$, PTCH, Gli1, Gli2, NGF, TrkA, p75, SP and CGRP 
were purchased from Abcam, USA. Recombinant sHH was obtained from R\&D Systems (Minneapolis, MN).

\section{Real-time PCR}

Total RNA was extracted using TRIzol (Invitrogen, CA, USA), and cDNA was synthesized using a Prime Script RT Reagent Kit (TaKaRa, Dalian, China). The real-time experiments were conducted on an iQ5 Multicolor Real-Time PCR Detection System (Bio-Rad, Hercules, CA) using an SYBR Green Real-time PCR Master Mix (TaKaRa, CA, USA). The primers are shown in online supplementary table 1 .

\section{Western blotting analysis}

Cells were lysed using a lysis buffer $(50 \mathrm{mM}$ Tris $(\mathrm{pH} 7.5)$, $150 \mathrm{mM} \mathrm{NaCl}, 1 \% \mathrm{NP}-40,0.5 \%$ sodium deoxycholate, $1 \mathrm{mM}$ EDTA, and $0.1 \%$ sodium dodecyl sulfate (SDS)) containing a protease inhibitor cocktail (Sigma-Aldrich), and protein concentrations were measured with the DC Protein Assay (BioRad Laboratories, Hercules, CA, USA). After separation on 7.5\% SDS-polyacrylamide gels, proteins were transferred to nitrocellulose membranes (Amersham Bioscience), which were then incubated with primary antibodies at $4^{\circ} \mathrm{C}$ overnight. After being washed three times with TBST, the membranes were incubated with horseradish peroxidase-conjugated secondary antibodies for 1 hour. Immunoreactive bands were visualized using an enhanced chemiluminescence kit (Millipore, MA, USA).

\section{ELISA}

To quantify the relative levels of pain factors in culture media and serum, an ELISA kit (R\&D, Minneapolis, MN, USA) was used according to the manufacturer's instructions.

\section{Plasmid construction and transfections}

Cells were seeded into small dishes and transfected with $100 \mathrm{nM}$ siRNA using Lipofectamine RNAi MAX Reagent (Invitrogen, CA, USA) according to the manufacturer's instructions. The cells were used for further experiments 24 hours after transfection. In addition, plasmids for the overexpression of sHH were constructed according to the manufacturer's instructions to study the activity of the sHH signaling pathway.

\section{Isolation and culture of mouse DRGs}

DRGs were isolated from newborn rats, stored on ice in DMEM:F12 (1:1) containing 20\% FBS, and then seeded into 24-well plates. The medium was changed every 3 days. DRGs were maintained at $37^{\circ} \mathrm{C}$ in a humidified atmosphere of $5 \% \mathrm{CO}_{2}$.

\section{Co-culture system for PC cells and DRGs}

When the DRGs were isolated from newborn rats, DRGs were subsequently seeded on 24-well plates in $25 \mu \mathrm{L}$ of an extracellular matrix (ECM) gel (BD Biosciences, Franklin Lakes, USA). The dishes were then placed in an incubator at $37^{\circ} \mathrm{C}$ with $5 \%$ $\mathrm{CO}_{2}$ for $30 \mathrm{~min}$ to enable the polymerization of the ECM gel, and then carefully submersed in $200 \mu \mathrm{L}$ DMEM supplemented with 10\% FBS. After 2-4 days, some axons from the DRGs

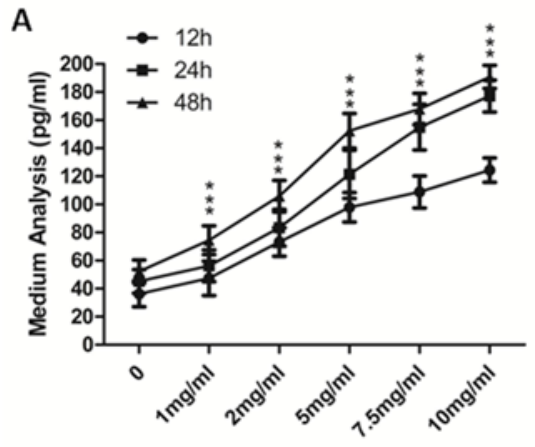

C

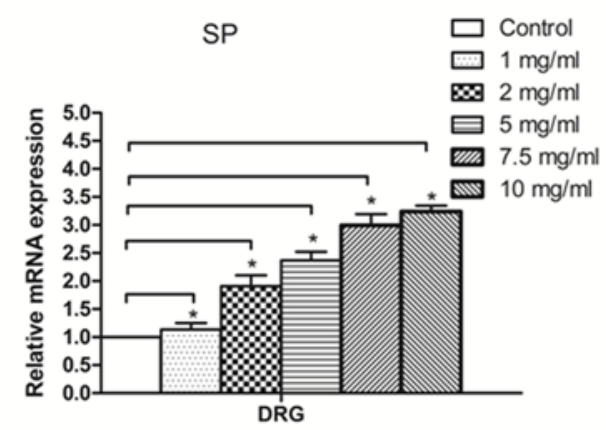

B

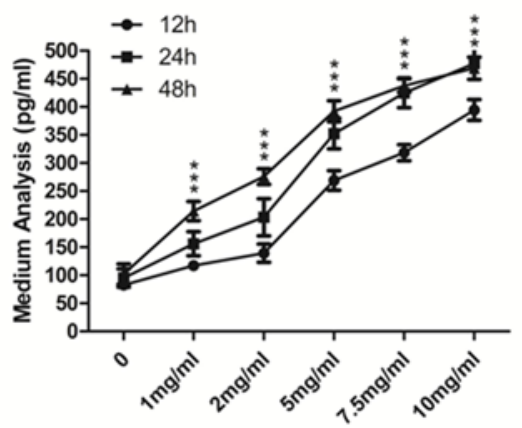

D

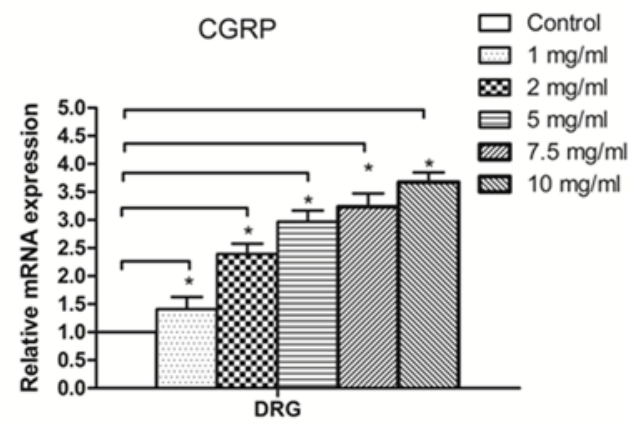

Figure 1 Sonic hedgehog $(\mathrm{sHH})$ increases the secretion and expression of SP and CGRP in DRGs. (A) SP secretion was analyzed in DRGs after treatment with different concentrations of sHH at 12, 24, and 48 hours. (B) CGRP secretion was analyzed in DRGs treated with different concentrations of sHH at 12,24, and 48 hours. (C) SP mRNA expression was determined in DRGs treated with different concentrations of sHH at 24 hours. (D) CGRP mRNA expression was determined in DRGs treated with different concentrations of sHH at 24 hours. The results showed that the secretion of SP and CGRP was dependent on the concentration and timing of $\mathrm{sHH}$. ${ }^{*} \mathrm{P}<0.05$ compared with control. All data from three independent experiments were analyzed. CGRP, calcitonin gene-related peptide; DRG, dorsal root ganglion; SP, substance P. 

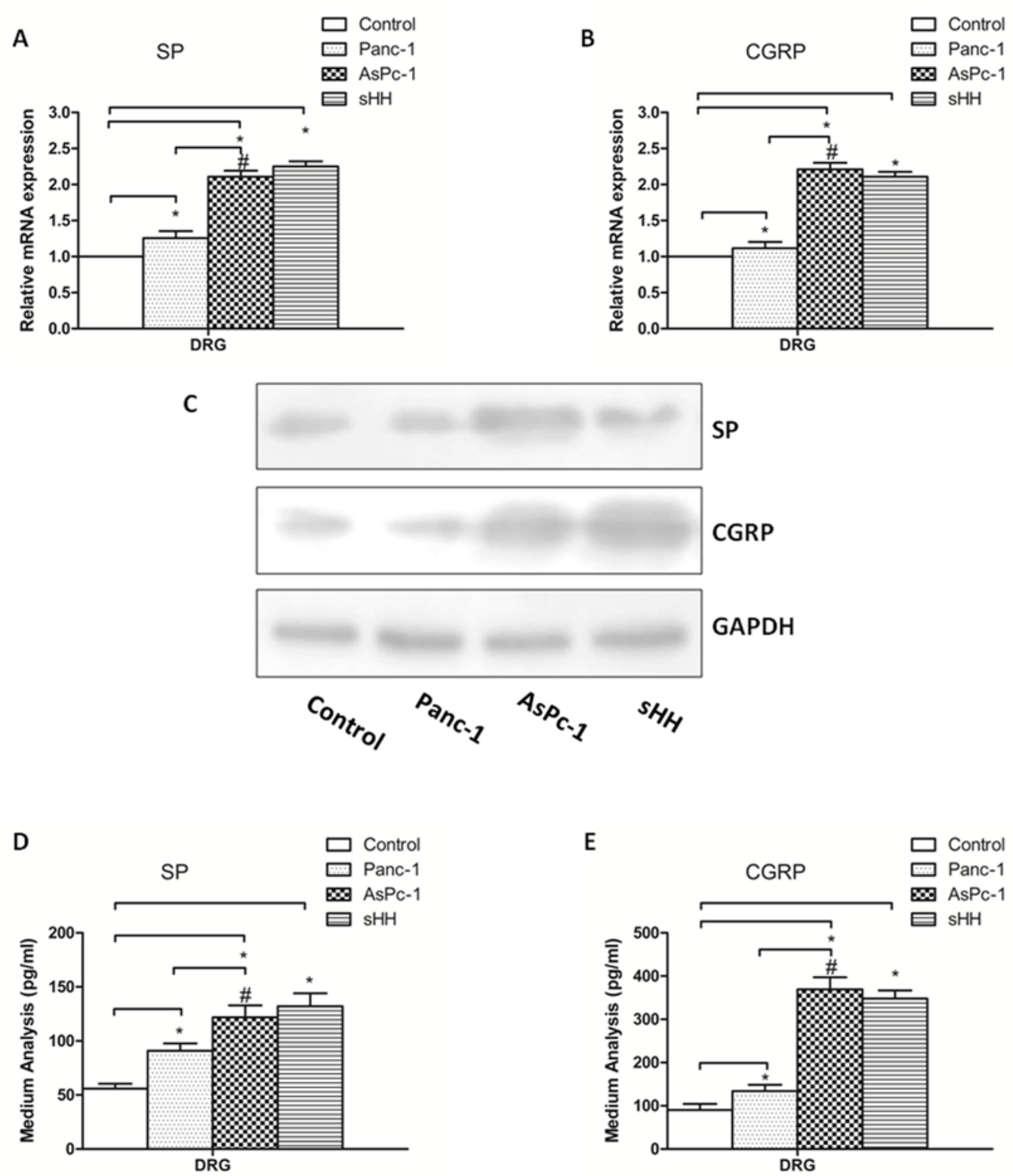

Figure 2 Co-culturing pancreatic cancer (PC) cells and DRGs increases secretion and expression of SP and CGRP in DRGs. (A, B) The mRNA expression of SP and CGRP was higher in AsPc-1 cells (high sHH expression) than in control and Panc-1 cells (low sHH expression). (C) The protein expression of SP and CGRP was regulated by PC cells. (D, E) Secretion levels of SP and CGRP were regulated by PC cells in DRGs. The results showed that protein expression and secretion results were similar to mRNA results. ${ }^{*} \mathrm{P}<0.05$ compared with control. \#P<0.05 between AsPc-1 and Panc- 1 cells. All data from three independent experiments were analyzed. CGRP, calcitonin gene-related peptide; DRG, dorsal root ganglion; sHH, sonic hedgehog; SP, substance P.

cultured. Then the medium was changed, and PC cells $(105 / \mu \mathrm{L}$, total $50 \mu \mathrm{L}$ ) were directly placed exactly $2 \mathrm{~mm}$ from the DRGs. The co-cultures were incubated at $37^{\circ} \mathrm{C}$ with $5 \% \mathrm{CO}_{2}$ in a humid atmosphere for 24 hours.

\section{Drug treatments}

In vitro, recombinant sHH was applied to DRGs at different concentrations for 12 hours. Cyclopamine, an sHH pathway inhibitor, was diluted to $18 \mu \mathrm{g} / \mathrm{mL}$ into DRG media for 12 hours. To detect the effect of neurotrophic factors, $60 \mathrm{ng} / \mathrm{mL}$ NGF was added into the DRG medium. GW441756 (15 $\mu \mathrm{M}$ for in vitro ${ }^{20}$, $30 \mathrm{mM}$ for in $\operatorname{vivo}^{21}$ ), an inhibitor of TrkA, was used to block TrkA effects in DRGs by addition into the media and by treatment of animals by intraperitoneal administration.

\section{Statistics}

Analysis was performed using the SPSS statistical software package (V.13.0). The significance of the data was determined using Student's t-test, one-way or two-way analysis of variance for in vitro and in vivo results. The significance level was set at $\mathrm{p}<0.05$. All results are presented as the mean \pm SD.

\section{RESULTS}

sHH increased SP and CGRP expression in DRGs

To investigate the possible relationship between $\mathrm{sHH}$ and pain factors in DRGs, we first evaluated how different concentrations of sHH affect DRGs. The mRNA and secretion levels of SP and CGRP in the culture medium were analyzed by 


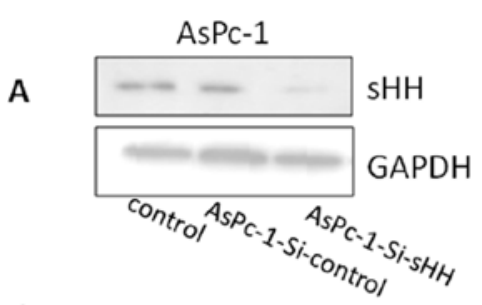

C

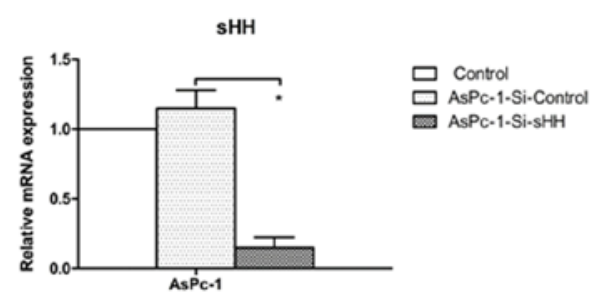

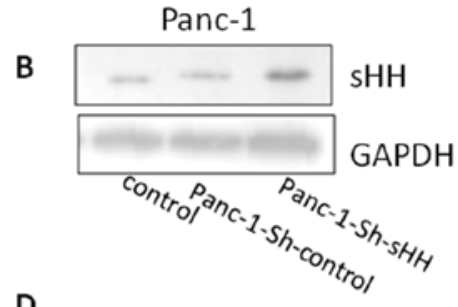

D

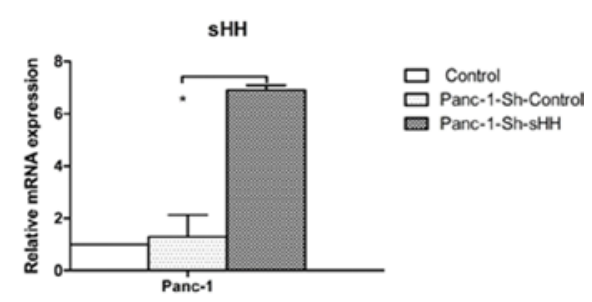

$E$

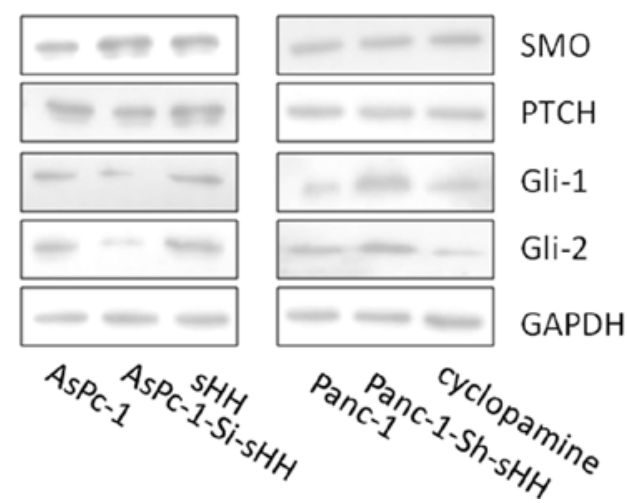

Figure 3 si-sHH-transfected pancreatic cancer (PC) cells activate the sHH signaling pathway in dorsal root ganglions (DRG). (A, B) The transfection efficiencies were verified by western blotting. Panc-1 cells (low sHH expression) were transfected with sHH plasmids, and AsPc-1 cells (high sHH expression) were transfected with sHH siRNA. (C, D) The transfection efficiencies were verified by real-time PCR. (E) sHH pathway signaling molecules in DRGs were checked by western blotting. The results showed that overexpressing sHH in Panc-1 cells induced the expression of the transcription factors Gli1 and Gli2, and knockdown of sHH in AsPc-1 cells using siRNA inhibited the expression of target molecules. ${ }^{*} \mathrm{P}<0.05$ compared with control. sHH, sonic hedgehog.

ELISA and real-time PCR (figure 1). The results show that the secretion of SP and CGRP was dependent on the duration and concentration of $\mathrm{sHH}$ treatment (figure $1 \mathrm{~A}, \mathrm{~B})(\mathrm{p}<0.05)$. Moreover, the mRNA results were similar to the ELISA results (figure $1 \mathrm{C}, \mathrm{D})(\mathrm{p}<0.05)$. Thus, the primary dose-effect relationship between $\mathrm{sHH}$ and pain factors was detected. In the following experiments, sHH was used at $5 \mathrm{mg} / \mathrm{mL}$ for 24 hours.

\section{Co-culture of sHH-expressing DRGs and PC cells increased SP and CGRP expression}

To determine whether PC cells mediate pancreatic pain by sHH, Panc-1 (low sHH expression) and AsPc-1 cells (high sHH expression) were used in our study. The expression and secretion of SP and CGRP were measured by ELISA, real-time PCR and western blotting in the co-culture system (PC cells were directly co-cultured with DRGs for 24 hours) (figure 2).

The mRNA results showed that the expression levels of SP and CGRP in DRGs were much higher in the co-cultured PC cells and $\mathrm{sHH}$ group than in the control group (figure $2 \mathrm{~A}, \mathrm{~B})(\mathrm{p}<0.05)$. Additionally, there was a significant difference between the Panc- 1 and AsPc-1 co-culture groups $(\mathrm{p}<0.05)$. To supplement the mRNA analyses, western blotting was used to quantify protein levels, and the results showed that protein expression was similar to mRNA expression (figure $2 \mathrm{C})(\mathrm{p}<0.05)$. Additionally, the concentrations of SP and CGRP in the culture medium were analyzed by ELISA. The results showed that the secretion of pain factors was much greater in the co-culture and sHH groups (figure $2 \mathrm{D}, \mathrm{E})(\mathrm{p}<0.05)$.

\section{sHH from PC cells activated the sHH signaling pathway in DRGs}

To determine whether sHH secretion from PC cells could activate the $\mathrm{sHH}$ signaling pathway in DRGs, the PC cell lines Panc-1 (low sHH expression) and AsPc-1 (high sHH expression) were transfected (figure 3). The transfection efficiency was checked by real-time PCR and western blotting (figure 3A-D). Moreover, we measured the expression of $\mathrm{sHH}$ pathway signaling molecules in DRGs by western blotting in the co-culture system (figure $3 \mathrm{E}$ ). The results showed that overexpressing sHH in Panc-1 cells induced the expression of the transcription factors Gli1 and Gli2 compared with control and cyclopamine-treated cells $(\mathrm{p}<0.05)$. In addition, knockdown of sHH in AsPc-1 cells using siRNA inhibited the expression of target molecules $(\mathrm{p}<0.05)$.

\section{Activation of the sHH signaling pathway increased the expression of NGF, P75, and TrkA in DRGs}

To determine whether the activation of the $\mathrm{sHH}$ signaling pathway significantly contributes to the induction of NGF and receptors in DRGs, we measured the expression of NGF, P75, 
A

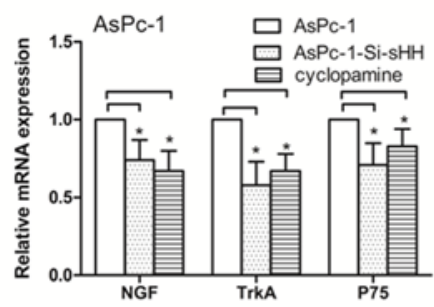

B

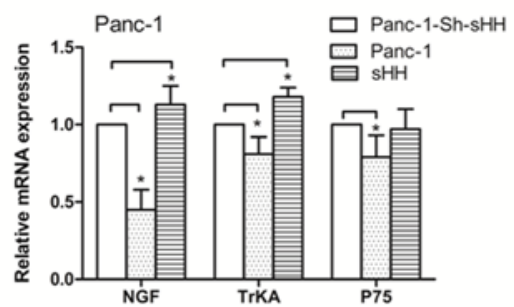

C

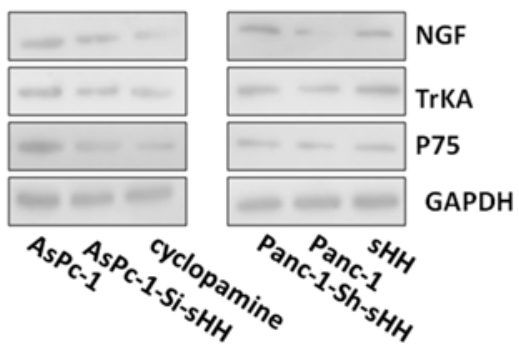

Figure 4 SHH signaling pathway activity increases the expression of NGF, P75 and TrkA in dorsal root ganglions (DRG). (A, B) The expression of NGF, P75 and TrkA in DRGs was checked by real-time PCR. The results demonstrated that NGF, P75 and TrkA were increased in DRGs co-cultured with pancreatic cancer (PC) cell lines that overexpress sHH. The expression of these molecules could be reversed by cyclopamine or siRNA against sHH. (C) The expression of NGF, P75 and TrkA in DRGs was checked by western blotting. ${ }^{*} \mathrm{P}<0.05$ compared with control. NGF, nerve growth factor; sHH, sonic hedgehog.

and TrkA in the co-culture system using real-time PCR and western blotting (figure 4). The mRNA results showed that NGF, P75, and TrkA were increased in DRGs co-cultured with sHHoverexpressing PC cell lines. Furthermore, the expression of NGF, P75, and TrkA could be reversed by treatment with cyclopamine or $\mathrm{sHH}$ siRNA (figure 4A,B) $(\mathrm{p}<0.05)$. Similar results were obtained with PCR and protein analysis by western blotting (figure 4C). Thus, activation of the $\mathrm{sHH}$ signaling pathway induced the expression of NGF and receptors in DRGs.

\section{sHH increased SP and CGRP expression in DRGs in an NGF- dependent manner}

To determine whether NGF contributed to the expression and secretion of SP and CGRP in the co-culture system, we quantified the expression levels of SP and CGRP in DRGs and in the medium by western blotting and ELISA, depending on the different groups (figure 5). The results showed that treatment with GW441756, an inhibitor of TrkA, alone or in combination with cyclopamine diminished the expression of SP and CGRP compared with control (figure 5A) $(\mathrm{p}<0.05)$.

The results showed the greatest inhibitory action in the combined group in AsPc-1 cells (figure 5B,C) $(\mathrm{p}<0.05)$. However, there was no difference between AsPc-1 cells transfected with si-sHH and those treated with GW441756 ( $\mathrm{p}>0.05)$. Additionally, in Panc-1 cells, the inhibitory effect of GW441756 was stronger than that of the other treatments/conditions (figure 5D,E) $(\mathrm{p}<0.05)$.

\section{sHH and NGF/NGF receptor determined pain sensitivity in a nude mouse model}

To determine whether $\mathrm{sHH}$ and $\mathrm{NGF} / \mathrm{NGF}$ receptor mediate PC-associated pain behavior in a mouse model, we designed an in situ tumor model in nude mice. PC cells were injected into the pancreas of nude mice. After 4 weeks, we measured the sensitivity of the abdomen to mechanical stimulation by the von Frey filament probing method. In addition, we quantified the secretion of SP and CGRP in the sera of each group of mice
2 hours after mechanical stimulation (figure 6). The frequency of response to mechanical stimulation for mice injected with NGF was greatest, and injection with GW441756 decreased the response to stimulation (figure 6C,D) $(n=10)(p<0.05)$. Panc-1 cells transfected with sh-sHH led to a greater sensitivity than Panc-1 control cells, and AsPc-1 cells transfected with si-sHH led to weaker sensitivity than those treated with NGF control. ELISA analysis revealed that the concentrations of SP and CGRP were consistent with the pain behavior results (figure 6A,B) $(\mathrm{p}<0.05)$.

\section{DISCUSSION}

PC is one of the most aggressive solid tumors, and sHH levels are elevated in human PC compared with normal pancreatic tissue. ${ }^{22}$ PNI extending into the extrapancreatic nerve plexus is a histopathological characteristic of PC and leads to abdominal pain. ${ }^{6}$ NGF induces thermal and mechanical sensitization of neurons, and the recent use of NGF-sequestering antibodies attests to the ongoing role of this neurotrophin in chronic pain. ${ }^{23}{ }^{24}$ However, the specific mechanism contributing to PC pain is unclear.

Previous studies have found that $\mathrm{sHH}$ is abnormally expressed in $\mathrm{PC}$ and is associated with cancer development and progression. ${ }^{10}$ Furthermore, inhibition of $\mathrm{sHH}$ signaling is an attractive clinical target for therapeutic intervention. ${ }^{25}$ In addition, our preliminary experiment demonstrated that the $\mathrm{sHH}$ signaling pathway contributed to PNI and PC pain by pancreatic stellate cells (PSC). ${ }^{26}$ In the current study, we first showed that the expression and secretion of SP and CGRP were dependent on the timing and concentration of sHH activity in DRGs. Then, we explored $\mathrm{sHH}$ expression in PC cells, where this ligand acts in a paracrine manner on DRGs to increase activation of sHH signaling. This is a new model of cancer-nerve interaction involving $\mathrm{sHH}$, nerve cells, and NGF in PC pain. In this study, we also showed that $\mathrm{sHH}$ increased the expression of NGF in an sHH-dependent manner in DRGs. The results demonstrated that NGF, P75, and TrkA were increased in DRGs co-cultured with sHH-overexpressing PC cell lines. Additionally, the increased 
A
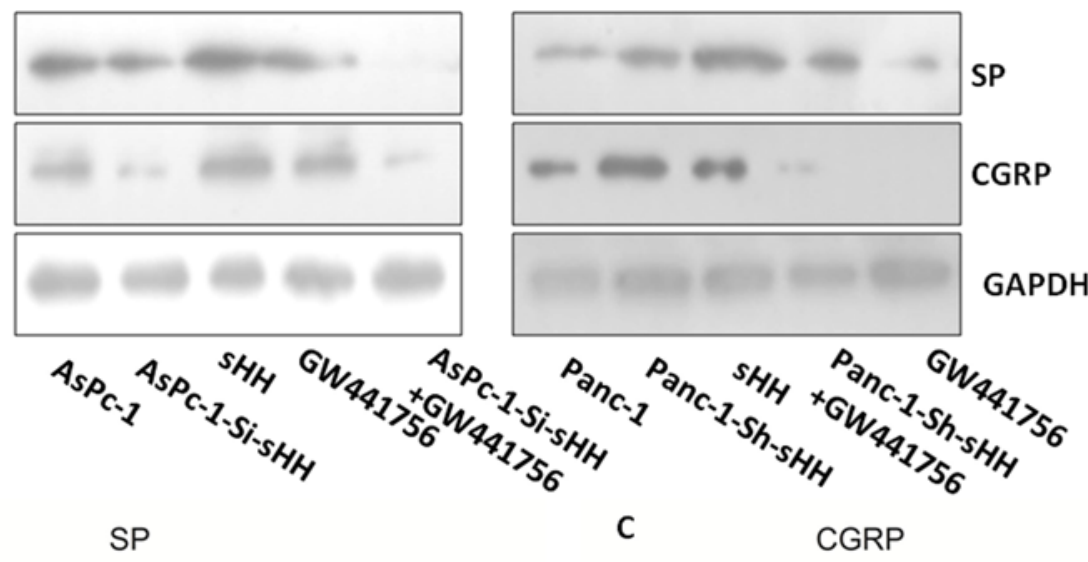

B
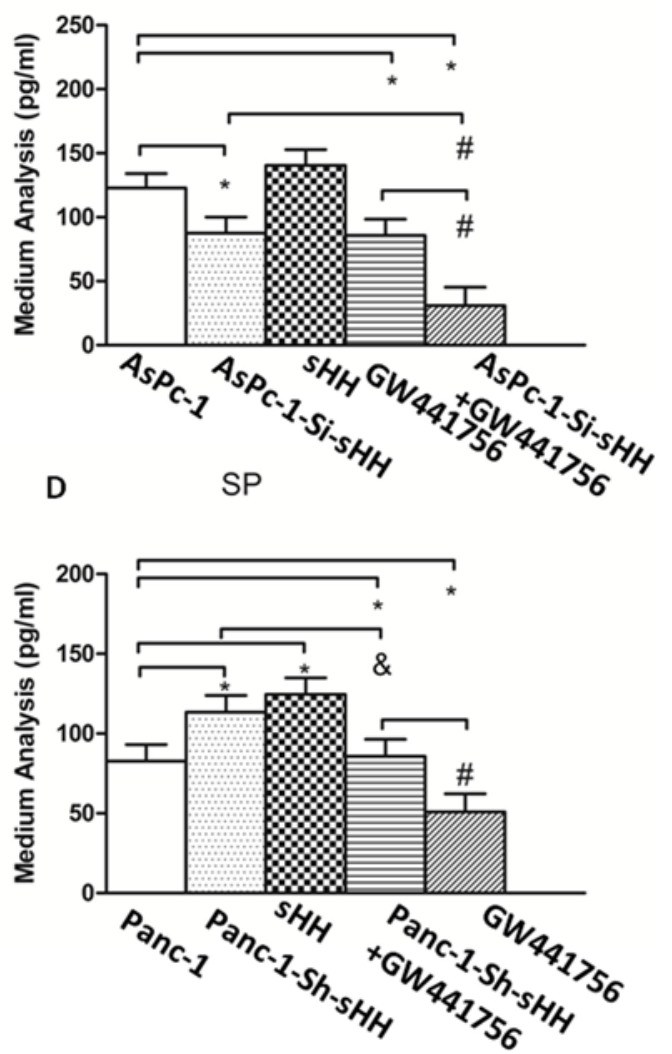
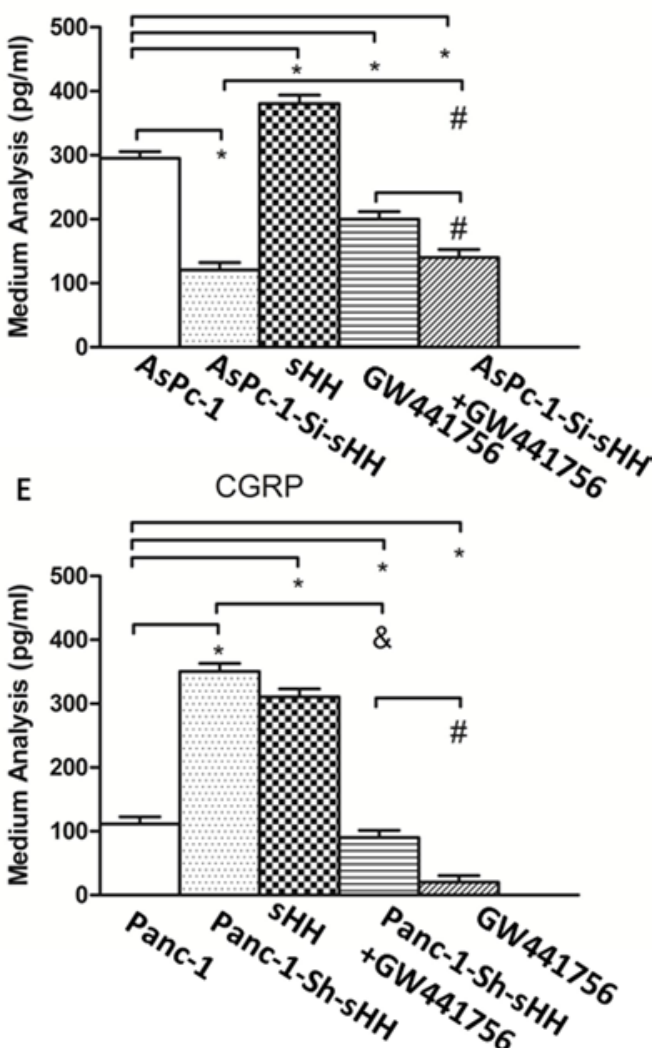

Figure 5 Nerve growth factor (NGF) and sHH increase the expression and secretion of SP and CGRP in dorsal root ganglions (DRG). (A) The expression levels of SP and CGRP were checked by western blotting. The results showed that GW441756, alone or in combination with cyclopamine, diminished the expression of SP and CGRP. (B, C) The secretion of SP and CGRP was determined by ELISA in the co-culture system containing AsPc1 cells and DRGs. ${ }^{*} P<0.05$ compared with control. \#P<0.05 compared with AsPc-1-Si-sHH+GW441756. (D, E) The secretion of SP and CGRP was determined by ELISA in the co-culture system containing Panc-1 cells and DRGs. ${ }^{*} P<0.05$ compared with control. \#P $<0.05$ between GW441756 and Panc-1-Sh-SHH+GW441756. \&P $<0.05$ between Panc-1-Sh-sHH and Panc-1-Sh-sHH+GW441756. All data from three independent experiments were analyzed. CGRP, calcitonin gene-related peptide; $5 H H$, sonic hedgehog; SP, substance P.

expression of NGF, P75, and TrkA could be reversed by treatment with cyclopamine or si-sHH.

Recent studies have suggested that the tumor microenvironment plays a considerable role in cancer cell proliferation, malignant progression, and response to therapy. ${ }^{27}$ Therefore, the interaction between the tumor stroma and PC cells may represent a novel therapeutic option for PC. ${ }^{28}$ Indeed, our previous results showed that PSCs played an essential role in the pain mechanism in PC. ${ }^{26}$ Briefly, sHH secreted from PC cells activates the $\mathrm{sHH}$ signaling pathway in PSCs and enables the complex pain process via NGF and brain-derived neurotrophic factor upregulation or sensitization to TRPV1 and pain factors in sensory nerves.

In previous studies, ${ }^{26}$ we discovered that PSCs contribute to neuropathic pain but do not play an indispensable role in the mechanism. Thus, we focused on the direct relationship between PC cells and DRGs through the sHH signaling pathway. The results confirmed that our hypothesis was feasible and that NGF was involved in neuropathic pain. The results also showed that an inhibitor of TrkA, alone or combined with cyclopamine, diminished the expression of SP and CGRP. 

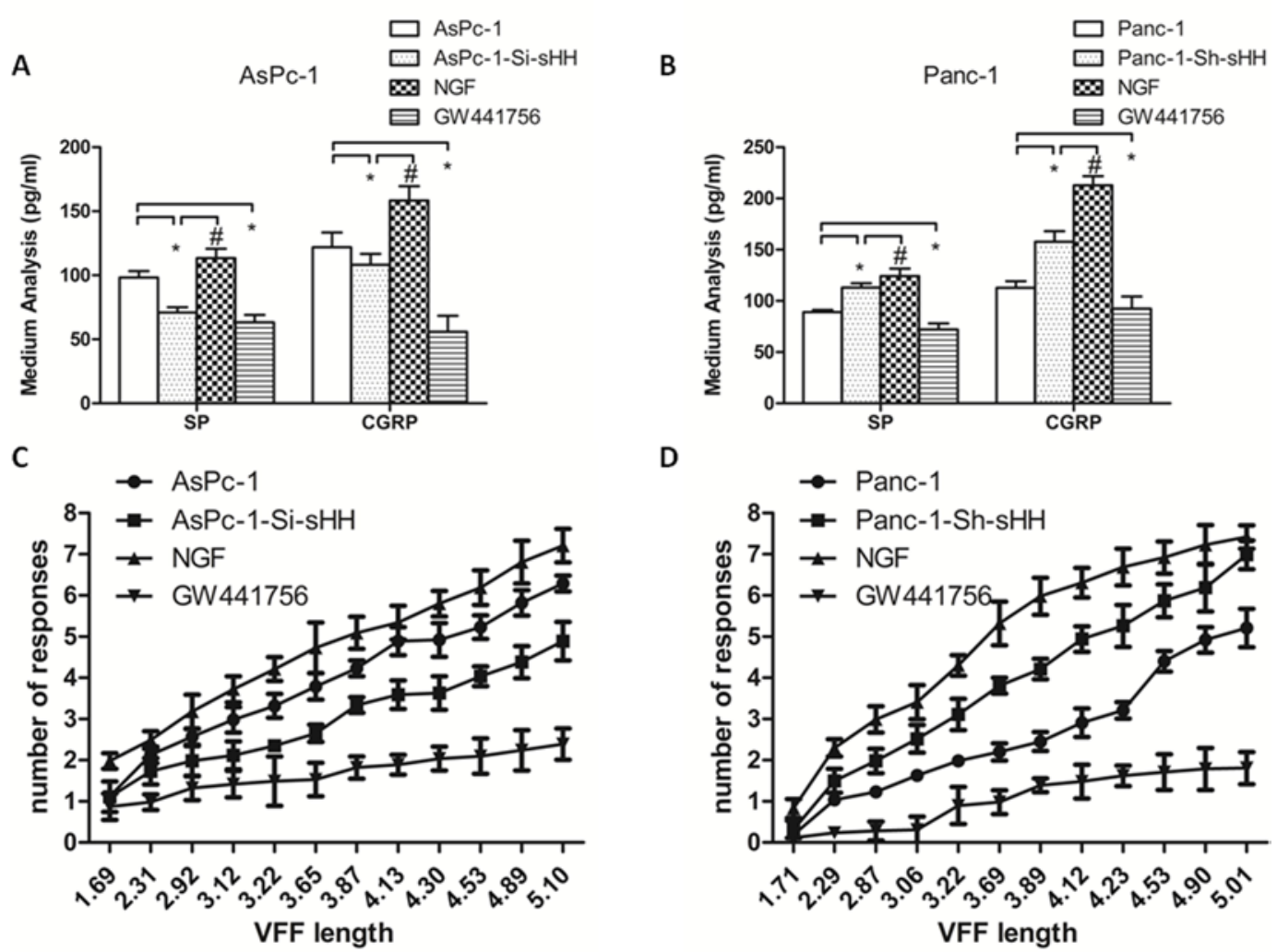

Figure 6 Sonic hedgehog $(\mathrm{sHH})$ and NGF aggravate pain behaviors and induce the secretion of SP and CGRP in mice. (A, B) NGF treatment and overexpression of sHH in pancreatic cancer (PC) cells resulted in greater pain sensitivity and higher secretion of SP and CGRP. However, GW441756 treatment and downregulation of sHH in PC cells decreased the secretion of SP and CGRP in the co-culture system. ${ }^{*} \mathrm{P}<0.05$ compared with control. $\# P<0.05$ between NGF and AsPc-1-Si-sHH group (for A); or between NGF and Panc-1-Sh-sHH (for B). (C, D) The response rate was the greatest in the NGF group. The weakest response was observed in the AsPc-1-si-sHH group. All data from three independent experiments were analyzed. CGRP, calcitonin gene-related peptide; NGF, nerve growth factor; SP, substance P; VFF, von frey filament.

The mechanical interactions between the tumor microenvironment and cancer pain are complicated and involve multiple factors. The sHH signaling pathway is not the only factor connecting PC cells and DRGs to contribute to neuropathic pain. Thus, despite the limitations of our research, this work contributes to the understanding of cancer-related pain and highlights areas that require further study.

In addition, the DRG was regarded as a whole in this study, and we did not differentiate the cell components. Schwann cells (SC), which are equally present in visceral nerves, were exposed to the hallmark components of the PC microenvironment. ${ }^{29} 30$ SC activation could decrease pain sensation in patients with PC and decrease mechanical hyperalgesia in mice with PC due to concomitant suppression of spinal astrocytes and microglia. ${ }^{29}$ Further follow-up experiments are needed to clarify the detailed mechanism of PC pain.

NGF is known to activate and promote the growth of sensory neurons. NGF and its interaction with its cognate receptor TrkA, a common marker of peptidergic neurons together with CGRP in the DRG, have come to be recognized as important mediators of pain. ${ }^{19}$ Our study provides evidence that NGF plays an important role in $\mathrm{PC}$ pain through regulation of $\mathrm{sHH}$ signaling. Thus, these findings suggest that NGF and TrkA are important for the generation and control of neuropathic pain.

In this study, an orthotopic tumor model was used to examine the experimental hypothesis in vivo. Von Frey filament testing ${ }^{31}$ showed a significant increase in pain behavior in the NGF and $\mathrm{sHH}$ groups. The results demonstrated that $\mathrm{sHH}$ signaling and
NGF are involved in pain generation in the animal model, consistent with the results of our previous in vitro studies. ${ }^{26}$ However, the mechanism of neuropathic pain is complex, and many other elements may be involved. Whether the SP and CGRP changes are mediated by different factors remains unknown. Thus, our results illuminate a path for further study.

\section{CONCLUSIONS}

In conclusion, sHH secreted from PC cells can increase the expression of NGF and TrkA by activating the $\mathrm{sHH}$ signaling pathway in DRGs. Additionally, the downstream pain factors induce expression changes in response to $\mathrm{sHH}$ and NGF. Our findings strengthen the rationale for PC-associated pain generation and may provide evidence for a novel therapeutic option for cancer pain.

Correction notice This article has been corrected since it publishe Online First. The second author affiliation has been amended.

Contributors Design: LH and QM. Writing: LH and JM. Analysis: JJ. Methodology: TQ, YX and MX. Data curation: EW and XS. All authors read and approved the final manuscript.

Funding This study was supported by the National Natural Science Foundation of China $(81502074,81672434,81702916)$.

Competing interests None declared.

Patient consent for publication Not required.

Ethics approval The Ethical Committee of the First Affiliated Hospital of Xi'an Jiaotong University. 
Provenance and peer review Not commissioned; externally peer reviewed.

Data availability statement Data are available in a public, open access repository. There are no data in this work. Data are available upon reasonable request. Data may be obtained from a third party and are not publicly available. All data relevant to the study are included in the article or uploaded as supplementary information.

Author note Corresponding author: Dr Qingyong Ma, Department of Hepatobiliary Surgery, Xi'an Jiaotong University Medical College First Affiliated Hospital, Xi'an, China;qyma56@ xjtu. edu. cn; Dr Jiguang Ma, Department of Anesthesiology,Xi'an Jiaotong University Medical College First Affiliated Hospital, Xi'an, China;jgma86@ mail.xjtu.edu.cn

Open access This is an open access article distributed in accordance with the Creative Commons Attribution 4.0 Unported (CC BY 4.0) license, which permits others to copy, redistribute, remix, transform and build upon this work for any purpose, provided the original work is properly cited, a link to the licence is given, and indication of whether changes were made. See: https://creativecommons.org/ licenses/by/4.0/.

\section{REFERENCES}

1 Siegel RL, Miller KD, Jemal A. Cancer statistics, 2019. CA Cancer J Clin 2019;69:7-34

2 Chen W, Zheng R, Baade PD, et al. Cancer statistics in China, 2015. CA Cancer J Clin 2016;66:115-32.

3 Lahoud MJ, Kourie HR, Antoun J, et al. Road map for pain management in pancreatic cancer: a review. World J Gastrointest Oncol 2016;8:599-606.

4 Bapat AA, Hostetter G, Von Hoff DD, et al. Perineural invasion and associated pain in pancreatic cancer. Nat Rev Cancer 2011;11:695-707.

5 Liang D, Shi S, Xu J, et al. New insights into perineural invasion of pancreatic cancer: more than pain. Biochim Biophys Acta 2016;1865:111-22.

6 Demir IE, Friess H, Ceyhan GO. Neural plasticity in pancreatitis and pancreatic cancer. Nat Rev Gastroenterol Hepatol 2015;12:649-59.

7 Yu H-M, Wang Q, Sun W-B. Silencing of FKBP51 alleviates the mechanical pain threshold, inhibits DRG inflammatory factors and pain mediators through the NFkappaB signaling pathway. Gene 2017;627:169-75.

8 Lu Z, Dong T-H, Si P-R, et al. Continuous low-dose-rate irradiation of iodine-125 seeds inhibiting perineural invasion in pancreatic cancer. Chin Med J 2016;129:2460-8.

9 Bapat AA, Munoz RM, Von Hoff DD, et al. Blocking nerve growth factor signaling reduces the neural invasion potential of pancreatic cancer cells. PLoS One 2016:11:e0165586.

10 Petrova E, Matevossian A, Resh MD. Hedgehog acyltransferase as a target in pancreatic ductal adenocarcinoma. Oncogene 2015;34:263-8

11 Thayer SP, di Magliano MP, Heiser PW, et al. Hedgehog is an early and late mediator of pancreatic cancer tumorigenesis. Nature 2003;425:851-6.

12 Song L, Liu Y, Yu Y, et al. Shh signaling guides spatial pathfinding of raphespinal tract axons by multidirectional repulsion. Cell Res 2012;22:697-716.
13 Denk F, Bennett DL, McMahon SB. Nerve growth factor and pain mechanisms. Annu Rev Neurosci 2017;40:307-25.

14 Bannwarth B, Kostine M. Targeting nerve growth factor (NGF) for pain management: what does the future hold for NGF antagonists? Drugs 2014;74:619-26.

15 Eskander MA, Ruparel S, Green DP, et al. Persistent nociception triggered by nerve growth factor (NGF) is mediated by TRPV1 and oxidative mechanisms. J Neurosci 2015;35:8593-603

16 Severini C, Petrocchi Passeri P, Ciotti MT, et al. Nerve growth factor derivative NGF61/100 promotes outgrowth of primary sensory neurons with reduced signs of nociceptive sensitization. Neuropharmacology 2017;117:134-48.

17 Ceyhan GO, Schäfer K-H, Kerscher AG, et al. Nerve growth factor and artemin are paracrine mediators of pancreatic neuropathy in pancreatic adenocarcinoma. Ann Surg 2010;251:923-31.

18 Demir IE, Friess H, Ceyhan GO. Nerve-cancer interactions in the stromal biology of pancreatic cancer. Front Physiol 2012;3:97.

19 Wang K, Demir IE, D'Haese JG, et al. The neurotrophic factor neurturin contributes toward an aggressive cancer cell phenotype, neuropathic pain and neuronal plasticity in pancreatic cancer. Carcinogenesis 2014;35:103-13.

20 Latina V, Caioli S, Zona C, et al. Impaired NGF/TrkA signaling causes early ADLinked presynaptic dysfunction in cholinergic primary neurons. Front Cell Neurosci 2017:11:68.

21 Hsieh Y-L, Kan H-W, Chiang H, et al. Distinct TrkA and RET modulated negative and positive neuropathic behaviors in a mouse model of resiniferatoxin-induced small fiber neuropathy. Exp Neurol 2018:300:87-99.

22 Kamisawa T, Wood LD, Itoi T, et al. Pancreatic cancer. Lancet 2016;388:73-85.

23 Kang J-Y, Yoo DY, Lee K-Y, et al. Sp, CGRP changes in pyridoxine induced neuropathic dogs with nerve growth factor gene therapy. BMC Neurosci 2016;17:1.

24 Bannwarth B, Kostine M. Nerve growth factor antagonists: is the future of monoclonal antibodies becoming clearer? Drugs 2017;77:1377-87.

25 Ko AH, LoConte N, Tempero MA, et al. A phase I study of Folfirinox plus IPI-926, a hedgehog pathway inhibitor, for advanced pancreatic adenocarcinoma. Pancreas 2016:45:370-5.

26 Han L, Ma J, Duan W, et al. Pancreatic stellate cells contribute pancreatic cancer pain via activation of sHH signaling pathway. Oncotarget 2016;7:18146-58.

27 Masamune A, Yoshida N, Hamada S, et al. Exosomes derived from pancreatic cancer cells induce activation and profibrogenic activities in pancreatic stellate cells. Biochem Biophys Res Commun 2018:495:71-7.

28 Neesse A, Algül H, Tuveson DA, et al. Stromal biology and therapy in pancreatic cancer: a changing paradigm. Gut 2015;64:1476-84.

29 Demir IE, Tieftrunk E, Schorn S, et al. Activated Schwann cells in pancreatic cancer are linked to analgesia via suppression of spinal astroglia and microglia. Gut 2016:65:1001-14

30 Demir IE, Kujundzic K, Pfitzinger PL, et al. Early pancreatic cancer lesions suppress pain through CXCL12-mediated chemoattraction of Schwann cells. Proc Natl Acad SCi U S A 2017;114:E85-94.

31 Hoogerwerf WA, Gondesen K, Xiao S-Y, et al. The role of mast cells in the pathogenesis of pain in chronic pancreatitis. BMC Gastroenterol 2005;5:8. 